ORIGINAL ARTICLE

\title{
Identification and characteristics of victims of violence identified by emergency physicians, triage nurses, and the police
}

\section{A Howe, M Crilly}

See end of article for authors' affiliations

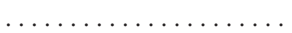

Correspondence to: Dr Andy Howe, East Lancashire Public Health Network, 33 Eagle Street, Accrington BB5 1LN, UK; andyhowe@clara.co.uk

\begin{abstract}
Objectives: The objectives of the study were threefold - to evaluate the identification and characteristics of victims of assault who attend an accident and emergency (A\&E) department; to compare the total number of assaults recorded in the A\&E department with the number recorded by the police; and to assess a system for collecting the location and method of assault.

Setting: The A\&E department of Chorley and South Ribble Hospital Trust, Lancashire, England.

Methods: A three month prospective study was performed. Victims of violence recorded on computer by doctors at discharge were compared with those identified at initial nurse triage. A comparison of police data with the A\&E data relating to Chorley residents was performed. Additional information on the method and location of assault was also collected.

Results: During the period $305(2.6 \%)$ of the patients attending A\&E were identified as having been assaulted. Of the 305 individuals, $236(77 \%)$ were identified by a doctor while $173(57 \%)$ such patients were identified by a triage nurse. A\&E identified twice the number of assaults involving Chorley residents as the police. Both men and women were most likely to have been injured on the street ( $44 \%$ and $37 \%$ respectively), although a greater proportion of women were injured at home $(24 \%$ ) than men (10\%). The majority of injuries were sustained by blows from fists, feet, and heads $(73 \%)$. Conclusions: A\&E doctors identify significantly more patients as the victims of violence than do nurses at triage. Using A\&E data identifies assaulted individuals not identified by the police. Computer systems can be used in A\&E to provide a more complete picture of the occurrence of violence in the community.
\end{abstract}

$\mathrm{T}$ he important impact that violence has upon health is reflected in the fact that in the United Kingdom the

National Health Service is now a partner, with police and local government, in the prevention of violence. ${ }^{\prime}$ Crime surveys and police data appear to seriously underestimate the level of violence in a community, ${ }^{2}$ and it has been suggested that information from accident and emergency (A\&E) departments on violent assault could assist in the development and evaluation of local preventative strategies. ${ }^{3}{ }^{4}$ In the United Kingdom, although data from A\&E departments has been used to describe the local epidemiology ${ }^{5}$ and trends in community violence, ${ }^{6}$ this information is not routinely available. In the United States, A\&E data have been more actively used in the development of weapon related injury surveillance programmes. ${ }^{7}$ The effective use of A\&E data requires that victims of violence are identified and relevant information is recorded. Basic violence surveillance data have previously been found to be incomplete in the paper clinical notes of both emergency department physicians and nurses. ${ }^{8}$ The use of clinical computerised systems has the potential for aiding data recording and retrieval, but the optimal method for collecting such data in A\&E has yet to be established. ${ }^{4}$

The aim of this study was to evaluate the identification of assaulted individuals who attend A\&E, by comparing victims initially identified at nurse triage with those identified by A\&E physicians before discharge from the A\&E department. A further comparison was made between the total number of assaults recorded in A\&E with the number recorded by the police over the same time period. A system of collecting the location and method of assault, information not normally available on the A\&E computer, was also assessed.

\section{METHODS}

This study was performed in a district general hospital A\&E department in Lancashire that records about 44000 patient visits per year, and serves a mixed urban and rural population. Information was collected prospectively on victims of assault recorded as such by either the nurse or doctor over a three month period (1 April 1999 to 30 June 1999).

"Assault" can be routinely entered onto the A\&E computer system as the patient's reason for attendance at the stage of nurse triage. Before a patient is discharged from the A\&E department, the attending doctor directly enters details of any injury, treatment, and outcome on to the clinical computer system. An additional A\&E computer screen template was set up to permit "doctor identified" assaulted patients to be recorded as such. A\&E physicians were required to record every patient attending A\&E between 1 April to 30 June 1999 as either "assaulted" or "not assaulted". Patients could not be discharged home from A\&E or transferred to another hospital specialist without completion of this additional computer template.

Additional questions concerning violent assault were also added to the computer screen template to identify the method of assault (body part, blunt object, glass, bottle, or knife).

The A\&E receptionist completed a separate computer template relating to the location of assault (home, street, public house, club or bar, work, school, someone else's home, sporting venue, or "other").

Abbreviations: $\mathrm{A} \& \mathrm{E}$, accident and emergency; $\mathrm{Cl}$, confidence interval; GP, general practitioner 


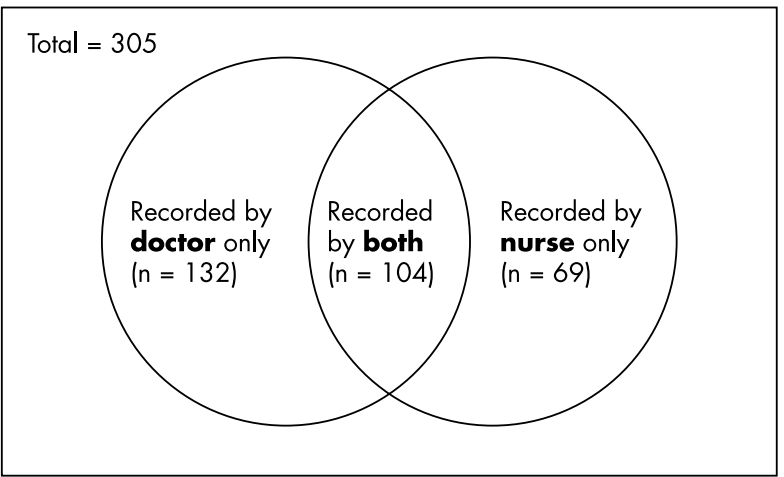

Figure 1 Venn diagram of patients identified by A\&E doctors and triage nurses as violently assaulted over three months at Chorley Hospital A\&E department, Lancashire, UK ( $n=305)$.

The A\&E clinical computer system was used to generate a list of all patients identified as "assault", by both triage nurse and A\&E physicians, over the study period. This list contained a unique personal identifier (A\&E episode number), name, age, date of birth, postcode, and time and date of attendance. The A\&E episode number was used to print a copy of the computer generated general practitioner (GP) letter (a summary letter routinely sent to GPs containing the computer held clinical details). Relevant information relating to the assault was extracted and directly entered into a Microsoft Access database.

Only one method of assault was analysed for each individual. The method of assault was defined in the order of priority as: knife (1); glass (2); bottle (3); blunt object (4); body part (5); bitten/scratched (6). The "priority" was applied only if a patient had been the victim of more than one method of assault.

For each victim of violence attending A\&E the post office directory was used to match a patient's home postcode to the electoral ward of residence.

The number of violent assaults recorded by the police over the same three month period was obtained for all 22 electoral wards of the district of Chorley (six urban and 16 rural wards). Assaults recorded by the police included the categories of "wounding and endangering life", "other wounding", "assault on a constable", and "common assault". Analysis was restricted to Chorley residents since Chorley A\&E department provides the nearest centre for emergency care for local residents injured locally. Residents of other neighbouring districts may travel to other local emergency departments.

\section{RESULTS \\ Identification of assault by A\&E doctors and triage nurses}

The total number of patients identified and recorded as violently assaulted during the three month study was 305, of whom 104 (34\%) were identified by both doctor and nurse (fig 1). The 305 patients identified as victims of assault represents $2.6 \%$ of all the patients attending Chorley A\&E ( 11 861) during the three month study period.

A total of $236(77 \%)$ patients were identified by A\&E doctors, while only $173(57 \%)$ patients were identified by a triage nurse (a difference in proportion of 20\%; 95\% confidence interval (CI) $13 \%$ to $27 \%$ ). Of the 305 patients identified, $225(74 \%)$ were male and $80(26 \%)$ were female. Categorised by age group, $56(18 \%)$ of the patients were aged under 16, 114 (37\%) aged 16-24, and 134 (44\%) were 25 years or older (age unavailable for one patient).

\section{Comparison of assaulted patients identified by doctors and triage nurses}

There were no significant differences in the characteristics of the assaulted patients identified by nurses and A\&E physi- cians. The ages of patients identified by either nurses or doctors were not significantly different (median ages 22 years and 23 years respectively; Mann-Whitney $U$ test, $\mathrm{p}=0.86$ ). Some $172(73 \%)$ of those identified by a doctor were male, while $123(71 \%)$ of those identified by nurses were male.

\section{Location of assault}

The location of assault was recorded by A\&E receptionists for all 305 patients. The commonest location for assault was the street $(43 \%)$ for both men $(44 \%)$ and women $(37 \%)$. Some $14 \%$ of all violent assaults occurred in the home; $10 \%$ in a public house, club or bar; $10 \%$ at work or school; $7 \%$ in someone else's home; $6 \%$ at a sporting venue; $11 \%$ in an "other" location. A significantly greater proportion of women (24\%) than men ( $10 \%)$ were assaulted at home (difference $14 \%, 95 \%$ CI $4 \%$ to $24 \%$ ). A greater proportion of men $(56 \%)$ were assaulted in a public place (street, public house, club, or bar) than women $(44 \%)$, although this difference was not statistically significant (difference $12 \% ; 95 \%$ CI $-1 \%$ to $25 \%$ ). Assault at a sporting venue, although relatively uncommon, was similar for both males and females.

There was little relationship between age and the location of assault, although $25 \%$ of the assaults to children under 16 occurred at school.

\section{Method of assault}

Of the 236 assaulted patients identified by A\&E doctors, information on the method of assault was available for 196 $(83 \%)$. Some 29 patients $(15 \%)$ had multiple methods of assault recorded ( 23 male; 27 over the age of 16). The majority of injuries $(143,73 \%)$ were inflicted by fists, feet, and heads while the remaining injuries were caused by blunt objects: 29 $(15 \%)$, bottles $11(6 \%)$, glass five $(3 \%)$, and being bitten or scratched four $(2 \%)$. Knives were recorded as being used in only four $(2 \%)$ cases of assault. There were no important differences between the sexes or between age groups in the main method of assault.

\section{A\&E versus police identification of assault}

Of the 305 patients identified as victims of assault over the three month period, 190 (62\%) lived within the Chorley district. In comparison with these 190 assaulted Chorley residents identified in A\&E, the police recorded only 96 assaults on Chorley residents over the same three month period. The proportion of assaults recorded by the police compared to those identified in A\&E was similar for both urban and rural electoral wards ( $51 \%$ and $50 \%$ respectively).

\section{DISCUSSION}

\section{Study findings}

This study has evaluated a method of data capture in A\&E and shown that doctors identify significantly more assaulted patients than do nurses at triage. While questionnaires have been developed to collect violence data from A\&E, ${ }^{9}$ no previous studies have evaluated the differences between triage nurses and doctors in the identification of victims of assault.

Although patient identifiable police data was unavailable for data protection reasons, the A\&E department recorded twice the number of victims of assault as the police. The reasons for this under-reporting by the police include the inability to identify the assailant, fear of reprisals by victims, and in the area of domestic violence an ongoing relationship with the assailant. ${ }^{6}$

\section{A\&E computer systems}

The identification of victims of assault using routinely collected computer data was limited by inherent restrictions of the clinical A\&E computer system used.

The incorporation of a specific computer template for assault into routine $A \& E$ practice for the three month study 
period was required to ensure the data capture of all doctor identified patients. Limitations of the computer system restricted both the number of actual questions and their format to a yes/no response. The questions selected were prioritised locally (for example gun related violence was excluded because it is a very rare event locally). The study was restricted to three months as this was a new approach to recording of assault in a busy A\&E department. This is in contrast with clinical computer systems in primary care which are well developed to capture such information. ${ }^{10}$

\section{Comparison with other studies}

The proportion of patients attending A\&E identified as victims of assault in this study (2.6\%) is similar to other studies in the United Kingdom $\left(2.4 \%{ }^{11}\right.$ and $\left.2.9 \%{ }^{12}\right)$, although our proportion of female victims $(26 \%)$ is higher than in previous studies $\left(20 \%{ }^{11}\right.$ and $\left.16 \%{ }^{12}\right)$. In contrast to our findings, the two previous United Kingdom studies both show that males were most likely to be assaulted on the street, while the majority of females were assaulted at home. ${ }^{11}{ }^{12}$

The high proportion of individuals attacked with fists, feet, or heads (73\%) in our study is similar to that reported in Bristol $(72 \%),{ }^{12}$ but higher than in Strathclyde $(43 \%) .{ }^{11}$ In this study, glass was involved in 3\% of assaults, bottles in 6\%, and knives in 2\%. In Strathclyde a "penetrating sharp" was used in $23 \%$ of attacks and glass in $15 \%$, while in Bristol knives were used in $6 \%$ of assaults. Such differences highlight the importance of developing local violence surveillance systems for targeting preventive strategies. Violence is a social and cultural issue and the spectrum of violence varies between countries. The extent of gun violence in the United States makes comparisons with the United Kingdom difficult. ${ }^{13}$

\section{Identification of victims}

While there is no "gold standard" identification test that allows us to know the true number of victims of assault, it is apparent that establishing a more accurate picture of victims of violent assault attending an A\&E will require information gathered from both A\&E nurses and physicians using a clinically responsive system. A computerised A\&E department allows the rapid collation of data routinely obtained as part of the health care process by highly trained professional staff (nurses, doctors, and receptionists). Since accuracy of information is important in medical record keeping (for medicolegal purposes), such information is likely to be of high quality. Although there is likely to be some variation in professionals' opinions, and in the diligence with which they collect and record data. ${ }^{14}$

For a victim of violence to be identified in A\&E involves the victim perceiving the event as an assault and actually disclosing the information to a health care professional..$^{15}$ Professional attitudes are likely to impact on identification, and the reasons for the differences in identification between nurses and physicians are almost certainly complex. The differences are probably partly due to the fact that the physician assessment is an inherently more detailed process than triage assessment. At triage victims of assault may simply be coded by nurses in relation to their injury (such as hand injury, wounds, or head injuries). Although doctors and nurses identified comparable groups of patients (by age and sex) as victims of violence, it is noteworthy that almost a quarter of cases were identified only by nurses at triage.

The identification of victims of violence is likely to be maximised by having both nurses and physicians directly inquire about the aetiology of injuries, a process that has been found to be acceptable to patients. ${ }^{16}$

\section{Violent injury databases}

Our findings reinforce the potential for using A\&E data to inform violence prevention. ${ }^{4}$ Our results indirectly support the

\section{Key points}

- At least $2.6 \%$ of patients attending A\&E departments have been violently assaulted.

- Only $34 \%$ of victims are identified by both triage nurses and $A \& E$ physicians. Differences in detection are not related to the patient's age or gender.

- Triage nurses identify $57 \%$ and A\&E physicians $77 \%$ of assaulted patients (a difference in proportion of 20\%; $95 \%$ Cl $13 \%$ to $27 \%$ )

- Only $50 \%$ of these assaults are recorded by the police with similar detection rates in urban and rural areas.

- Effective violence surveillance requires information from multiple sources to provide a comprehensive picture of violence in the community.

concept of multidisciplinary computerised violent injury databases that have been developed in the United States linking emergency medical services, hospital outpatient and inpatient records, coroner records, vital statistics (death certificates), police reports, and court records. ${ }^{7}$ Although our results relate to a single A\&E department, we suspect that similar results may be found elsewhere, and we hope that others will attempt to replicate our study.

\section{ACKNOWLEDGEMENTS}

We thank the staff of Chorley A\&E Department for their cooperation, and in particular, Dr Richard Fairhurst for his assistance and advice.

\section{Authors' affiliations}

A Howe, East Lancashire Public Health Network, Lancashire, UK M Crilly, Department of Public Health, University of Aberdeen, Scotland, UK

\section{REFERENCES}

1 Home Office. Crime \& Disorder Act 1998 introductory guide. London: Home Office, 1998

2 Shepherd J, Shapland M, Scully CS. Recording by the police of violent offences; an accident and emergency department perspective. Med Sci Law 1989:29:251-6.

3 Shepherd JP. Tackling violence. BM 1998;316:879-80.

4 Shepherd JP, Sivararjasingam V, Rivara FP. Using injury data for violence prevention. BM 2000;321:1481-2.

5 Gorman DR, Ramsay W, Wilson GS, et al. Using routine accident and emergency department data to describe local injury epidemiology. Public Health 1999;1 13:285-9.

6 Sivarajasingam V, Shepherd JP. Trends in community violence in England and Wales 1995-1998: an accident and emergency department perspective. Emerg Med J 2001;18:105-9.

7 Mercy JA, Ikeda R, Powell KE. Firearm-related injury surveillance. Am J Prev Med 1998;15(3S):6-16.

8 Houry D, Feldhaus KM, Nyquist SR, et al. Emergency department documentation in cases of intentional assault. Ann Emerg Med 1999:34:715-9.

9 Goodwin V, Shepherd JP. The development of an assault patient questionnaire to allow accident and emergency departments to contribute to Crime and Disorder Act local crime audits. J Accid Emerg Med 2000;17:196-8.

10 Hassey A, Gerrett D, Wilson A. A survey of validity and utility of electronic patient records in a general practice. BM 2001;322:1401-5

11 Wright J, Kariya A. Assault patients attending a Scottish accident and emergency department. J R Soc Med 1997;90:322-6.

12 Shepherd J. Violent crime in Bristol: an accident and emergency department perspective. Br J Criminol 1990;30:289-305.

13 Frattaroli S, Teret SP. Why firearm injury surveillance? Am J Prev Med $1998 ; 15(3 S): 2-5$

14 Lindsey K, Stevens $\mathrm{H}$. The role of the accident and emergency department. In: Bewley S, Friend J, Mezey G, eds. Violence against women. London: Royal College of Obstetricians and Gynaecologists Press, 1997.

15 Acierno R, Resnick HS, Kilpatrick DG. Health impact of interpersonal violence 1: prevalence rates, case identification, and risk factors for sexual assault, physical assault, and domestic violence in men and women. Behav Med 1997:23:53-64.

16 Howe A, Crilly M, Fairhurst R. Acceptability of asking patients about violence in accident and emergency. Emerg Med J 2002;19:138-40. 